\title{
DETECTION OF INSECT DAMAGED WHEAT KERNELS BY IMPACT ACOUSTICS
}

\author{
Tom C. Pearson ${ }^{1}$, A. Enis Cetin ${ }^{2}$,Ahmed H. Tewfik ${ }^{3}$ \\ USDA-ARS Manhattan, Kansas ${ }^{1}$ \\ Bilkent University, Ankara, Turkey ${ }^{2}$ \\ University of Minnesota, Minneapolis, $\mathrm{MN}^{3}$
}

\begin{abstract}
Insect damaged wheat kernels (IDK) are characterized by a small hole bored into the kernel by insect larvae. This damage decreases flour quality as insect proteins interfere with the bread-making biochemistry and insect fragments are very unsightly. A prototype system was set up to detect IDK by dropping them onto a steel plate and processing the acoustic signal generated when kernels impact the plate. The acoustic signal was processed by three different methods: 1) modeling of the signal in the time domain, 2) computing time domain signal variances in short time windows, and 3), analysis of the frequency spectra magnitudes. Linear discriminant analysis was used to select a subset of features and perform classification. 98\% of un-damaged kernels and $84.4 \%$ of IDK were correctly classified.
\end{abstract}

\section{INTRODUCTION}

Internal insect infestation of wheat kernels degrades quality and value of wheat and is one of the most difficult defects to detect. Insect infestation causes grain loss by consumption, contaminates the grain with excrement and fragments, causes nutritional losses, and degrades end-use quality of flour [1]. Wheat millers usually specify that wheat loads must contain less than five insect-damaged kernels (IDK) per $100 \mathrm{~g}$. Inspecting for IDK's is labor intensive and may miss most of the infested kernels where an immature insect has not emerged from the kernel.

Wheat kernels become infested when an adult female insect chews a small hole into the kernel, about $0.05 \mathrm{~mm}$ in diameter, deposits an egg, and then seals the egg with a mixture of mucus and the wheat that was chewed out. The egg plug is the same color as the wheat surface so it is nearly impossible to detect by external examination. When the egg hatches, the insect larvae develop and consume tunnels inside the wheat kernel until it reaches maturity. Finally, the insect exits the kernel by chewing an exit tunnel.
Several methods have been used, or are currently under development, to detect insect damage inside whole wheat kernels [1]. These include staining the egg plug to detect weevil infestation, flotation methods, $x$-ray imaging, acoustic detection of larval movement and chewing, carbon dioxide measurement, and staining of amino acids specific to insect body fluids. However, most of these methods have only achieved limited implementation either because they are slow, labor intensive, expensive, can only detect specific insect species, or cannot quantitatively measure insect infestation levels. More recently, an image analysis program has been developed to automatically scan x-ray images for insect infestation [2]. Other researchers have investigated use of near-infrared (NIR) spectroscopy to detect hidden insects in wheat kernels [3]. Finally a digital signal processing method has been developed to work in conjunction with a Perten SingleKernel Characterization System (SKCS) to detect insectdamaged kernels [4]. X-ray, NIR methods, and SKCS methods suffer from high false-positive error rates (good kernels classified as infested) and are still cost prohibitive for many commercial applications. Thus, no economically viable and simple equipment utilizing these technologies has yet become available for detection of internal insects.

It is proposed that IDK can be discriminated from undamaged kernels by means of impact acoustic emissions. Since IDK are hollowed out from the insect tunnel, it stands to reason that their impact acoustic emissions would be different than un-damaged kernels. However, these methods are not readily adaptable to high speed inspection. A high-throughput, low cost acoustical system for sorting pistachio nuts has been developed to separate pistachio nuts with closed shells from those with cracked shells [5-7]. In this system, pistachio nuts drop onto a steel plate and the sound of the impact is analyzed in real time. Pistachio nuts with closed shells produce a different sound than those with cracked shells, as expected. Classification accuracy of this system is approximately $97 \%$, with a throughput rate of approximately 40 nuts/second. The cost of the system is approximately $\$ 5000$ and it works reliably in a food processing environment with little maintenance or skill required to operate. Simple features such as amplitude of the sound, decaying rate of the sound 
amplitude, etc. were used to reach a decision to accept or reject the nut. More recently, Cetin et al. (2003) [6,7] developed an improved classification scheme for use in this sorter. This scheme utilized standard practices used in speech-recognition systems in which the sound feature vector consists of mel-cepstral coefficients along with principle components of magnitude of acoustic impact emission signals to identify closed- and open-shell nuts.

The objective of this study is to develop signal processing algorithms to show feasibility for real-time inspection of wheat infested with stored grain insects. The overall aim of the project is to detect, classify and remove contaminated and defective kernels using impact acoustic, or the sound created when a kernel strikes a steel plate, as this mode of acoustic excitation is easily adapted to high throughput sorting systems.

\section{MATERIALS AND METHODS}

A common stored grain insect, the rice weevil, was reared in kernels from hard red winter wheat (HRW) wheat. A total of 500 kernels with no insect damage and 500 kernels with an emerged hole were picked out by visual inspection and used in experimental apparatus.

Experimental apparatus: A schematic of the experimental apparatus (Figure 1) for singulating wheat kernels, dropping them onto the impact plate, then collecting the acoustic emissions from the impact are shown in Figure 1. The vibration feeder consisted of a steel trough in the shape of a "V" which conveyed the kernels from the bulk hopper into a single file by the time they reached the end of the feeder. The impact plate was a polished block of stainless steel approximately $7.5 \times 5.0 \mathrm{x}$ $10 \mathrm{~cm}$. The mass of the impact plate is much larger than the wheat kernels in order to minimize vibrations from the plate interfering with acoustic emissions from kernels.

Two microphones, which are sensitive to frequencies up to $100 \mathrm{KHz}$ are used in order to sense ultrasonic acoustic emissions from the wheat kernels. It was found that there can be an order of magnitude difference in intensity of the acoustic emissions among kernels. Thus, one microphone was amplified at $314 \mathrm{mV} / \mathrm{Pa}$ and the other at $31.4 \mathrm{mV} / \mathrm{Pa}$ to ensure that the system has the required dynamic range to capture the acoustic emissions. Microphone signals are digitized at a sampling frequency of $192 \mathrm{KHz}$ with 16 bit resolution. The data acquisition was triggered using an optical sensor. Upon receipt of a trigger signal, the computer would acquire 2000 data points from the microphone signal. After acquisition, the signal was first high pass filtered using a single pole recursive filter with a cutoff frequency of $9600 \mathrm{KHz}$. This filtering effectively eliminated $60 \mathrm{~Hz}$ noise, any DC offset, and eliminated effects from ambient sounds.

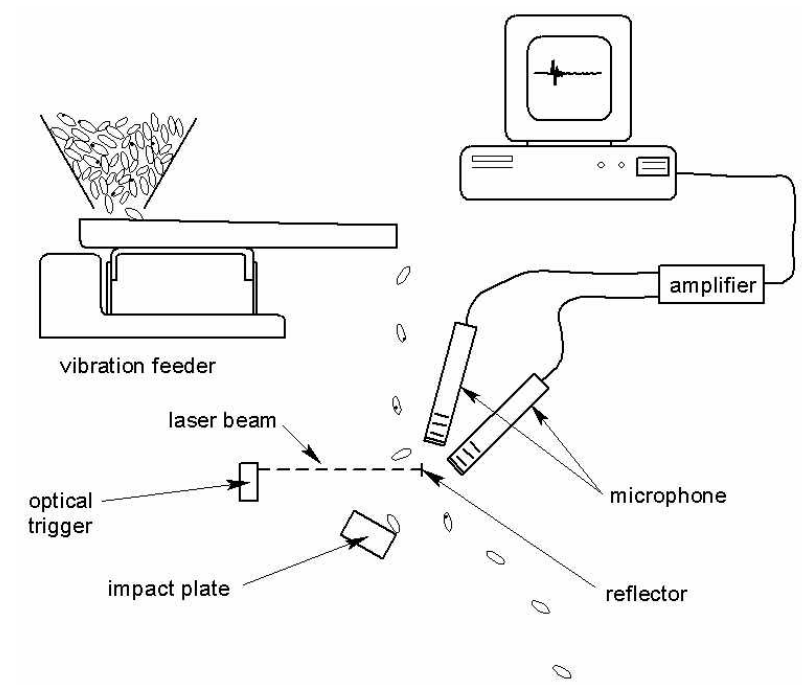

Figure 1. Schematic of experimental apparatus for collecting acoustic emissions from wheat kernels

2.1 Data processing: Features from the two microphones were obtained by the same processing. Subsequently, classification was performed using linear discriminant analysis (LDA) using features from each microphone independently. Finally, a kernel was classified as IDK only if the LDA from both microphones indicated it was IDK. Features were extracted from the signals by three different methods: 1) modeling of the signal in the time domain, 2) computing time domain signal variances in short time windows, and 3) analysis of the frequency spectra magnitudes. Each of these methods will be discussed separately below.

Time domain signal modeling: Typical signals from an un-damaged kernel and IDK are shown in Figure 2. The maximum amplitude of the signals is quite variable but the overall shape of signals emitted from IDK's and un-damaged kernels is more consistent. Damaged kernels have a strong tendency to resonate for a longer time than un-damaged kernels. This phenomenon is due to the insect tunnel within the body of the kernel. To characterize this type of signal response, we modeled the signal after transforming it in the following steps outlined below: (i) rectify the signal by taking the absolute value at all points, (ii) non-linearly filter the signal by replacing the center data point with the maximum value in a seven point window, and (iii) Non-linearly estimate the four parameters of the Weibull function, given by equation 1 , which has a shape similar to that of the processed time domain signal.

$$
Y=\frac{c b}{a}\left[\frac{\left(x-x_{o}\right)}{a}\right]^{(b-1))}\left\{e^{-\left[\frac{\left(x-x_{o}\right)}{a}\right]^{b}}\right\}
$$

where $x>x_{o}$, otherwise $Y=0$. 
The parameters $a, b, c$ and $x_{o}$ are parameters of the Weibull equation that are iteratively estimated by nonlinear regression dynamic link library (DLL) (NLREG Phil Sherod, Brentwood, TN), $Y$ is the magnitude of the Weibull function at data point $x$. The parameters $a$ and $b$ determine the shape of the resulting curve while parameters $c$ define the magnitude of the signal and $x_{o}$ determines where the Weibull curve begins. Parameter $a$ controls the width of the curve while $b$ controls its shape. When $b \approx 3.5$, the curve approximates a Gaussian, when $b \approx 2$, the curve approximates a log-normal curve, and when $b \approx 1$, the curve approximates an exponential decay. Thus, parameters $a$ and $b$ are potentially useful features for distinguishing IDK from un-damaged kernels irregardless of the signal magnitude. Additionally, the mean-square error value $R^{2}$ from the curve fitting can also aid in the identification of IDK. Figure 3 shows an example of signals that have been processed as described above and Table 1 shows average parameter estimates for all kernels.
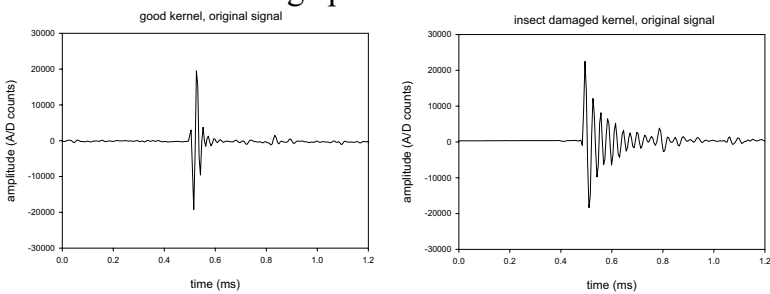

Figure 2. Typical signals from an un-damaged kernel (left) and IDK. Note that duration of the IDK is longer.
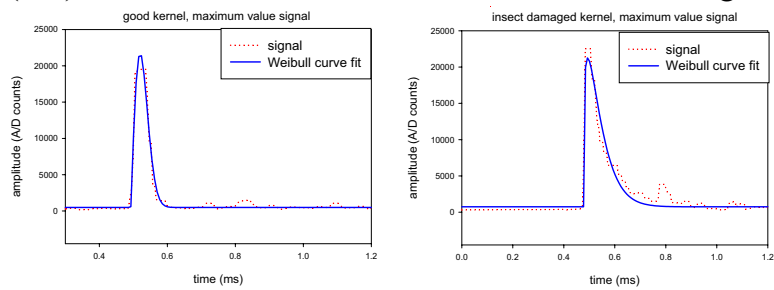

Figure 3. Maximum value filtered signals fitted with a Weibull curve for an un-damaged kernel (left) and IDK (right).

Table 1. Average Weibull parameter estimates.

\begin{tabular}{|c|c|c|c|c|}
\hline \multirow{2}{*}{ parameter } & \multicolumn{2}{|c|}{ Un-damaged } & \multicolumn{2}{c|}{ Insect damaged } \\
\cline { 2 - 5 } & avg & std dev & avg & std dev \\
\hline$a$ & 15.01 & 8.06 & 27.99 & 19.37 \\
\hline$b$ & 2.75 & 1.19 & 1.59 & 0.78 \\
\hline$R^{2}$ & 0.85 & 0.17 & 0.80 & 0.13 \\
\hline
\end{tabular}

Short time variance window processing: In addition to the time domain processing by modeling the signals with a Weibull function, variances of the signals are also computed in short time windows. Weibull function captures the shape of the recorded signal globally and the short-time variance information models the local time domain variations in the signal. The short time windows were 50 points in duration and incremented in steps of 30 points so that each window overlapped by 20 points. The first window began 40 points in front of the maximum signal magnitude. A total of eight short time windows were computed to cover the entire duration of all signals. After all variances were computed, they were normalized by the sum of all eight variances as follows

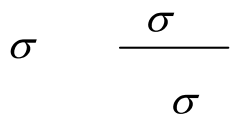

where $\sigma_{n i}^{2}$ and $\sigma_{i}^{2}$ are the normalized and computed variances from window $i$ with $i=1$ being the first window and $i=8$ being the last. This method captures the increased duration of the signals from insect damaged kernels. As can be seen from Figure 4, the average normalized variance of the third window is greater than that from undamaged kernels. Additionally, the slope between the third and fourth normalized variance is different.

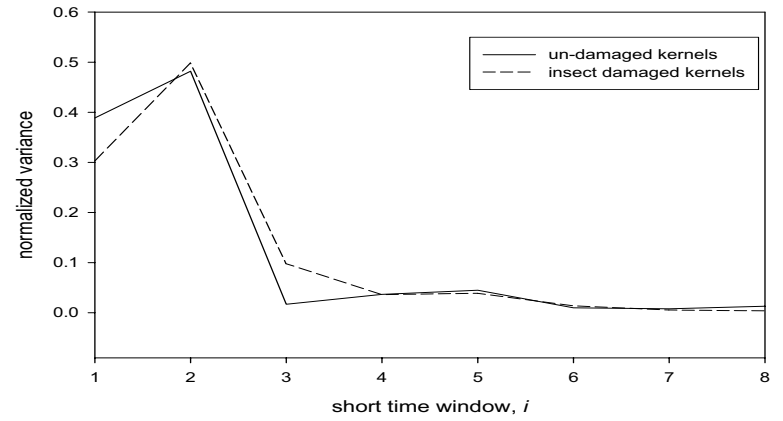

Figure 4. Average normalized variances from short time windows of time domain signals.

Frequency Domain Processing: A 256 point discrete Fourier Transform (DFT) was computed from each signal using a Hamming window. The 256-point window covers the impact sound of the wheat kernel and it starts about 80 data points before the signal maximum slope which corresponds to the impact moment of the kernel. The magnitude of each spectra was computed and then low pass filtered using a 20 tap FIR filter was applied to remove jagged spikes in the spectra. The low pass filter has a cutoff frequency of $\pi / 4$ in the normalized DFT domain. As can be seen in Figure 7, the frequency spectra of insect damaged kernels have a sharper peak that occurs at a lower frequency than the un-damaged kernel. Higher resolution DFT's show that IDK have two peaks, however, this feature is often lost in noise. In this example, peaks of the spectra of un-damaged kernels and IDK are clearly distinguishable but there are significant overlaps in many cases which are mainly due to the tumbling of the wheat kernel on the metal plate. The frequency corresponding to the peak magnitude in the frequency spectra was saved as a potential discriminating feature. In addition, the 15 magnitude values before the peak and 15 points after the 
peak were saved and normalized by the peak magnitude. For undamaged kernels, the average frequency corresponding to the peak DFT magnitude was $34.5 \mathrm{KHz}$ with a standard deviation of $9.1 \mathrm{KHz}$. In contrast, IDK's had an average peak frequency of $26.3 \mathrm{KHz}$ with a standard deviation of $9.6 \mathrm{KHz}$.

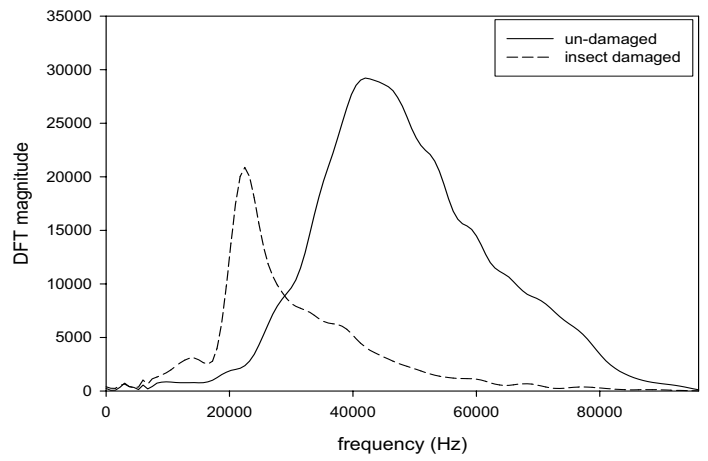

Figure 5. Example frequency spectra magnitudes for an undamaged and insect damaged kernel.

\section{CLASSIFICATION AND RESULTS}

The Weibull curve fit parameters $a, b$ and $R^{2}$, all eight normalized variances from the short time windows, the frequency corresponding to the peak DFT magnitude, and the 15 normalized DFT magnitudes before and after the peak DFT magnitude were pooled together as potential discriminating features. Stepwise discriminant analysis (significance for entry and exit $=0.01$ ) was used to select a small subset of these features for classification by discriminant analysis. The data sets were evenly divided into two randomly selected training and validation sets. Feature selection with stepwise discriminant analysis [8] followed by discriminant function computation was performed on the training sets, and then tested on the validation sets. Both pooled and non-pooled covariance matrices were tested. This feature selection and classification procedure was performed independently for each microphone signal. Finally, a kernel was classified as IDK only if the classification from both microphones indicated that it was IDK, otherwise the kernel was classified as good.

Using the above method, $98.0 \%$ of the un-damaged kernels and $84.4 \%$ of IDK were correctly classified. All types of features were selected: Weibull $a, b$, and $R^{2}$, short time window variances, frequency corresponding to the maximum DFT magnitude, and normalized DFT magnitudes about the peak magnitude. The stepwise feature selection procedure indicated that the Weibull parameters were the most significant for classification. Table 2 shows the classification accuracies when this method (stepwise selection, LDA, and OR logic on the two microphones) was applied to only a portion of the features extracted from the signal.
Table 2. Classification accuracies for subsets of features.

\begin{tabular}{|c|c|c|}
\hline \multirow{2}{*}{ Features } & \multicolumn{2}{|c|}{ Percent accuracy } \\
& Undamaged & IDK \\
\hline Weibull $a, b$, and $R^{2}$ & 88.8 & 86.6 \\
\hline Short time window variances & 85.2 & 76.2 \\
\hline DFT domain info. & 87.4 & 85.0 \\
\hline All features & 98.0 & 84.4 \\
\hline
\end{tabular}

\section{CONCLUSIONS AND FUTURE WORK}

This work shows that use of impact acoustic emissions for detection of IDK is a feasible and promising method. It is experimentally observed that more accurate classification results than manual inspection can be achieved by impact acoustical analysis in the data set. The computational cost of classifying a kernel is very low and as a result, it is possible to inspect large quantities of wheat kernels using the proposed technique. Using the algorithm presented here, the signal processing only required $20 \mathrm{~ms}$ to perform with the Weibull curve fitting taking about half this time.

Further experimentation and development is needed to expand this method using a wider range of wheat types grown under different climatic conditions. Additionally, data from other types of wheat defects (i.e. mold damage, cracks, etc) will be needed to determine if the presence of other defects can be discriminated from un-damaged kernels and IDK. Quite likely, further signal processing and a neural network will be needed to obtain high classification accuracies on more varied data.

\section{REFERENCES}

[1] Pederson, J. 1992. Insects: Identification, damage, and detection. In Storage of Cereal Grains, ed. D. B. Sauer. St. Paul, Minn.: American Association of Cereal Chemists.

[2] Haff, R. P. 2001. X-ray inspection of wheat for granary weevils. PhD diss. Davis, Calif.: University of California, Davis. [3] Dowell, F. E., J. E. Throne, and J. E. Baker. 1998. Automated nondestructive detection of internal insect infestation of wheat kernels by using near-infrared reflectance spectroscopy. J. of Economic Entomology 91(4): 899-904.

[4] Pearson, T.C., et.al 2003. Automated detection of internal insect infestations in whole wheat kernels using a Perten SKCS 4100. Applied Engineering in Agriculture. 19(6):727-733

[5] Pearson, T.C. 2001. Detection of pistachio nuts with closed shells using impact acoustics. Applied Engineering in Agriculture, 17(2):249-253.

[6] Cetin, A.E., T.C. Pearson, A. H. Tewfik, "Classification of closed- and open-shell pistachio nuts using voice-recognition technology," Transactions of the ASAE, 47(2), 659-664, 2004.

[7] Cetin, A.E., T.C. Pearson, A. H. Tewfik. 2004. Classification of closed- and open-shell pistachio nuts using impact acoustical analysis, presented in ICASSP 2004.

[8] Huberty, C. J. 1994. Applied Discriminant Analysis. New York: John Wiley and Sons, Inc. 